\title{
Electrochemical Behavior of Palladium-Containing Nanoparticles on a Carbon Electrode in Hydrochloric Acid Solutions
}

\author{
Alexander S. Romanchenko*, Maxim N. Likhatski, \\ Anton A. Karacharov and Yuri L. Mikhlin \\ Institute of Chemistry and Chemical Technology SB RAS \\ $F R C$ "Krasnoyarsk Scientific Center of the $S B$ RAS" \\ 50/24 Akademgorodok, Krasnoyarsk, 660036, Russia
}

Received 26.11.2018, received in revised form 29.11.2018, accepted 02.02.2019

The electrochemical behavior of metallic palladium nanoparticles obtained by reducing $\mathrm{H}_{2} \mathrm{PdCl}_{4}$ with formaldehyde and the products of the interaction of $\mathrm{H}_{2} \mathrm{PdCl}_{4}$ with sodium sulfide with a different ratio of reagents in a solution of hydrochloric acid was studied by the method of cyclic voltammetry. Voltammograms of metallic and sulfide nanoparticles were obtained, and interpretation was given to the observed electrochemical processes. The initial and oxidized systems with different potential values were characterized by X-ray photoelectron spectroscopy (XPS). It is shown that palladium sulfide nanoparticles, obtained at high molar ratios of reagents $(S / P d=3: 1)$, are more resistant to oxidative and reducing effects than those obtained at low molar ratios (S/Pd $=1: 1)$.

Keywords: electrochemistry, nanoparticles, palladium, sulfur, immobilization, carbon, cyclic voltammetry, X-ray photoelectron spectroscopy.

Citation: Romanchenko A.S., Likhatski M.N., Karacharov A.A., Mikhlin Yu.L. Electrochemical behavior of palladiumcontaining nanoparticles on a carbon electrode in hydrochloric acid solutions, J. Sib. Fed. Univ. Chem., 2019, 12(1), $42-53$. DOI: 10.17516/1998-2836-0107.

\footnotetext{
(C) Siberian Federal University. All rights reserved

* Corresponding author E-mail address: romaas82@mail.ru
} 


\title{
Электрохимическое поведение
}

\author{
палладийсодержащих наночастищ \\ на углеродном электроде \\ в солянокислых растворах
}

А.С. Романченко, М.Н. Лихацкий, А.А. Карачаров, Ю.Л. Михлин Институт химии и химической технологии СО РАН ФИЦ «Красноярский научный иентр СО РАН» Россия, 660036, Красноярск, Академгородок 50/24

\begin{abstract}
Методом ииклической вольтамперометрии изучено электрохимическое поведение металлических наночастии палладия, полученных путем восстановления $\mathrm{H}_{2} \mathrm{PdCl}$ формальдегидом и продуктов взаимодействия $\mathrm{H}_{2} \mathrm{PdCl}_{4}$ с сульфидом натрия, с различнымм соотношением реагентов в растворе соляной кислоты. Получены вольтамперограммы металлических и сульфидных наночастии, дана интерпретащия наблюдаюшимся электрохимическим процессам. Исходные и окисленные до различного значения потенциала системы охарактеризованы методом рентгеновской фотоэлектронной спектроскопии (РФЭС). Показано, что наночастицы сульфида палладия, полученные при высоких мольных соотношениях реагентов ( $S / P d=3: 1)$, болееустойчивы кокислительному ивосстановительному воздействию, чем полученные при низких мольных соотношениях ( $S / P d=1: 1)$.
\end{abstract}

Ключевые слова: электрохимия, наночастиць, палладий, сера, иммобилизация, углерод, ичиклиеская вольтамперометрия, рентгеновская фотоэлектронная спектроскопия.

\section{Введение}

Наночастицы в сравнении с объемными материалами имеют отличные свойства, как физические, так и химические [1-4]. С одной стороны, это открывает новые возможности в материаловедении, оптике и микроэлектронике, катализе [1-4]. С другой стороны, появление новых свойств может привести к неожиданным, либо нежелательным результатам. Палладий и его сульфиды широко применяются в катализе, а его наночастицы имеют перспективы применения в различных областях, например изготовление сенсоров [1-4]. К тому же при переработке руд и концентратов, содержащих платиновые металлы, значительная их часть переходит в раствор либо присутствует в сырье в виде нано- и субмикронных частиц, в том числе связанных с серой $[5,6]$. В данном случае происходит практически полная потеря платиновых металлов, связанных с серой, - они переходят в хвосты [7]. Дальнейшие пути перемещения платины и палладия не изучены несмотря на актуальность, в том числе и экономическую выгоду. Палладий имеет высокое сродство к сере и помимо обычных сульфидов образует также полисульфидные комплексы $[8,9]$, роль которых практически не учитывается при моделирова- 
нии поведения палладия в растворах, содержащих серу. Имеется множество работ, изучавших электрохимическое поведение металлических наночастиц и других наноразмерных объектов палладия [10-13]. Электрохимическое поведение наночастиц палладия, связанного с серой, не изучалось несмотря на актуальность данных исследований в области материаловедения и микроэлектроники (способы получения новых материалов с заданными свойствами), а также в гидрометаллургии для усовершенствования методов переработки палладийсодержащего сырья и установления механизмов и путей переноса палладия в природе и техногенных объектах. Цель работы - изучение электрохимического поведения наноразмерных частиц палладия, связанного с серой, полученных при взаимодействии тетрахлоропалладата (II) водорода с раствором сульфида натрия, взятых при разных мольных соотношениях.

\section{Экспериментальная часть}

Исходный раствор тетрахлоропалладата (II) водорода с концентрацией по металлу 0,19 М для подавления гидролиза стабилизировали 6 М соляной кислотой, концентрация рабочего раствора $\mathrm{Na}_{2} \mathrm{~S}$ составляла 0,1 M, гидроксида натрия - 0,1 М. Металлические наночастицы палладия получали следующим образом: при перемешивании с помощью магнитной мешалки в конической колбе к 33 мл раствора $\mathrm{H}_{2} \mathrm{PdCl}_{4}\left(0,3\right.$ ммоль/л) после нагрева до $85^{\circ} \mathrm{C}$ для частичной нейтрализации прибавляли 3,25 мл 0,1 моль/л раствора $\mathrm{NaOH}$, затем вносили 1 мл 0,05 моль/л раствора цитрата тринатрия, задав тем самым молярное отношение [цитрат $]_{0} /\left[\mathrm{H}_{2} \mathrm{PdCl}_{4}\right]_{0}=5: 1$, и сразу же прибавляли 67 мкл раствора формалина (38 \% формальдегида, <4 \% метанола). Нагрев и перемешивание реакционного раствора поддерживали в течение 2 ч. Для получения наночастиц сульфида палладия в конической стеклянной колбе при комнатной температуре к 30 мл 0,3 мМ водного раствора $\mathrm{H}_{2} \mathrm{PdCl}_{4}$ прибавляли расчетный объем раствора 0,1 М сульфида натрия так, чтобы задать определенные начальные соотношения $\left[\mathrm{Na}_{2} \mathrm{~S}_{0} /\left[\mathrm{H}_{2} \mathrm{PdCl}_{4}\right]_{0} \quad 3: 1\right.$ и $1: 1$, раствор слегка встряхивали, но не перемешивали. Реакционный раствор анализировали методом фотонной корреляционной спектроскопии с помощью прибора Zeta Sizer Nano (Malvern) и оптической спектроскопии (спектрометр Shimadzu 3600). В обоих случаях наблюдали изменение окраски до светло-коричневой; ширина запрещенной зоны в приближении непрямозонного перехода составляла 1,35 эВ в начале реакции и уменьшалась до $\approx 1,12$ эВ по истечении 3 ч после смешивания реагентов. Гидродинамический диаметр рассеивателей, по данным фотонной корреляционной спектроскопии, при молярном отношении реагентов 1:1 по истечении 3 ч от начала синтеза составлял 11 нм, в то же время присутствовали и более крупные рассеиватели с объемной долей не более 16 \% и размерами несколько мкм, которые можно отнести на счет гидроксополимеров. Частицы с гидродинамическим диаметром около 30 нм были единственной фракцией в случае синтеза при отношении $[\mathrm{S}]_{0} /[\mathrm{Pd}]_{0}$ 3:1.

Электрохимические исследования и окислительная обработка проводились на потенциостате P 30 SM, фирмы Elins (г. Москва). Для проведения экспериментов капля исследуемого золя наносилась на поверхность кусочка пирографита (марка ZYB) и высушивалась при комнатной температуре. После высыхания капли поверхность промывалась от электролитов, затем к образцу подводился электрический контакт и погружался в раствор 0,1 M HCl. После установления равновесия - компромиссного потенциала - примерно 400 мВ (в работе все потенциалы приводятся относительно насыщенного хлорсеребряного электрода) производилась развертка 
потенциала со скоростью 20 мВ/с в анодном, либо катодном направлении. По достижении заданного потенциала электрод вынимали из раствора и высушивали на воздухе. Рентгеновские фотоэлектронные спектры записывали на спектрометре SPECS при возбуждении немонохроматизированным излучением $\mathrm{Mg} \mathrm{K} \alpha$ анода рентгеновской трубки при энергии пропускания 20 эВ для обзорных спектров или 8 эВ (узкие сканы) полусферического энергоанализатора PHOIBOS 150 MCD9. Разложение спектров выполняли с помощью программного пакета CasaXPS после вычитания нелинейного фона по Ширли с использованием гауссовско-лоренцевской формы максимумов. Отношения элементов на поверхности определяли по обзорным спектрам с учетом эмпирических коэффициентов чувствительности.

\section{Результаты и обсуждение}

\section{1. Металлические наночастицы палладия}

Для изучения вольтамперного поведения $\mathrm{H}_{2} \mathrm{PdCl}_{4}$ на обновленную поверхность пирографита наносилась капля раствора (0,19 моль/л), высушивалась, после чего проводились электрохимические эксперименты. Как показано на рис. 1, нет принципиальных отличий между кривыми развертки потенциала начатых в анодном и катодном направлениях. На анодных ветвях можно видеть два пика. Первый имеет положение примерно при -200 мВ, а второй, широкий пик - при 650 - 800 мВ. На катодной ветви наблюдается один пик вблизи 0 мВ, он имеет относительно высокую интенсивность в первом цикле развертки, а во втором цикле его интенсивность резко снижается. Также в последующих циклах снижается интенсивность анодного пика при -200 мВ. На вольтамперной кривой, начатой в анодном направлении, наблюдается не широкий пик в области $800 \mathrm{MB}$, а более узкий, менее интенсивный и при большем значении потенциала - 890 мВ (рис. $1 a$ ).

Анализируя литературные данные [14-16] и полученные вольтамперные зависимости, пик 2 на катодной ветви в первом цикле (рис. $1 a$ и б) следует отнести к восстановлению палладия, входящего в состав хлоридного комплекса до металла.
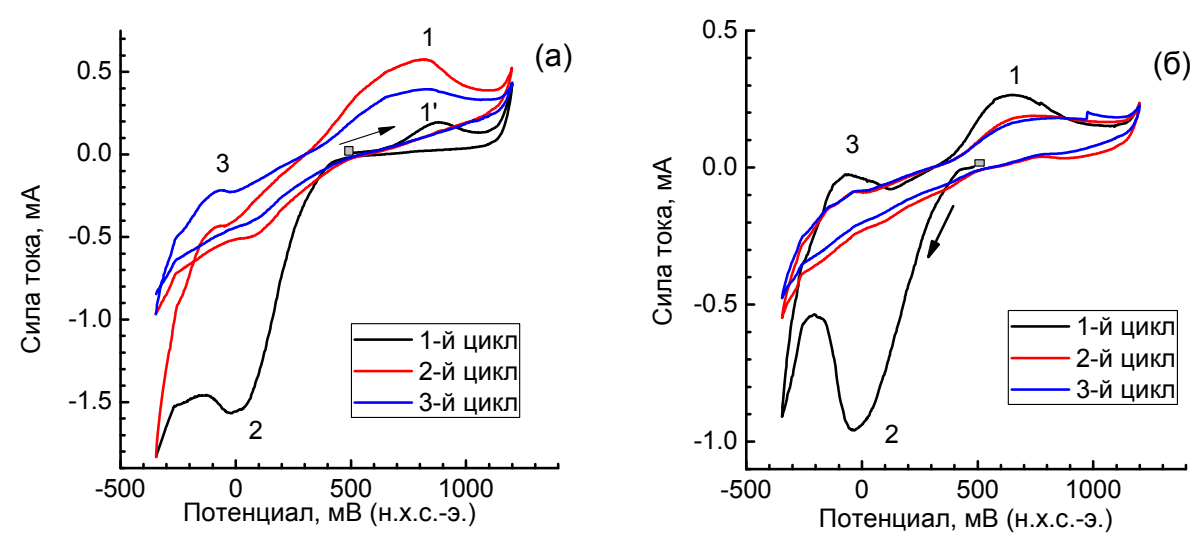

Рис. 1. Вольтамперные зависимости высушенной на пирографите капли водного раствора $0,19 \mathrm{M} \mathrm{H}_{2} \mathrm{PdCl}_{4}$. Скорость развертки потенциала $20 \mathrm{mB} / \mathrm{c}, 22{ }^{\circ} \mathrm{C}$. Направление развертки потенциала: а) анодное и б) катодное

Fig. 1. Current-voltage characteristics of a pyrographite-dried drop of an aqueous solution of $0.19 \mathrm{M} \mathrm{H}_{2} \mathrm{PdCl}_{4}$. The potential sweep speed is $20 \mathrm{mV} / \mathrm{s}, 2{ }^{\circ} \mathrm{C}$. The potential sweep direction: a) anodic and б) cathodic 


$$
\mathrm{PdCl}_{4}^{2-}+2 \mathrm{e}^{-} \rightarrow \mathrm{Pd}^{0}+4 \mathrm{Cl}^{-} .
$$

Частицы палладия, образующиеся при спонтанном осаждении на поверхности пирографита, имеют пик окисления при 880 мВ, что выше потенциала окисления палладия, образующегося при катодном восстановлении (650-820 мВ). Данное отличие может быть обусловлено размерным эффектом либо структурой частиц. Окисление металлического палладия, учитывая наличие в электролите хлорид-ионов, идёт с образованием комплексных ионов, которые переходят в объем раствора. С этим процессом связано снижение интенсивности практически всех пиков во втором и последующих циклах развертки потенциала.

\section{2. Металлические наночастицы палладия, полученные формальдегидным способом}

Пик 2 на анодной ветви отвечает восстановлению водорода, растворенного в решетке металлического палладия [17]. Восстановлению водорода на катодной ветви отвечает область резкого возрастания тока, при $\mathrm{E}<-250 \mathrm{MB}$. Пик 3 на анодной ветви при $800 \mathrm{MB}$ отвечает окислению металлического палладия до $\mathrm{PdCl}_{4}^{2-}$, реакция обратная (1). На вольтамперной кривой, начатой в анодном направлении (рис. 1a), пик 1' также отвечает окислению металлического палладия, который спонтанно восстанавливается из комплекса $\mathrm{H}_{2} \mathrm{PdCl}_{4}$ при его контакте с поверхностью пирографита, за счет окисления поверхностных форм углерода [18].

На рис. 2 приведены вольтамперные характеристики электрода с нанесенными наночастицами палладия, полученными смешением формальдегида с раствором $\mathrm{H}_{2} \mathrm{PdCl}_{4}$. На вольтамперной зависимости в первом цикле развертки потенциала на анодной ветви наблюдается пик при 660 мВ (рис. 2a), отвечающий окислению металлических наночастиц палладия. В последующих циклах интенсивность этого пика снижается на порядок. Пик 3, при -100 мВ, отвечающий окислению растворенного в палладии водорода, присутствует и во втором цикле, но его интенсивность очень мала, а в третьем цикле он практически не наблюдается (рис. 26).
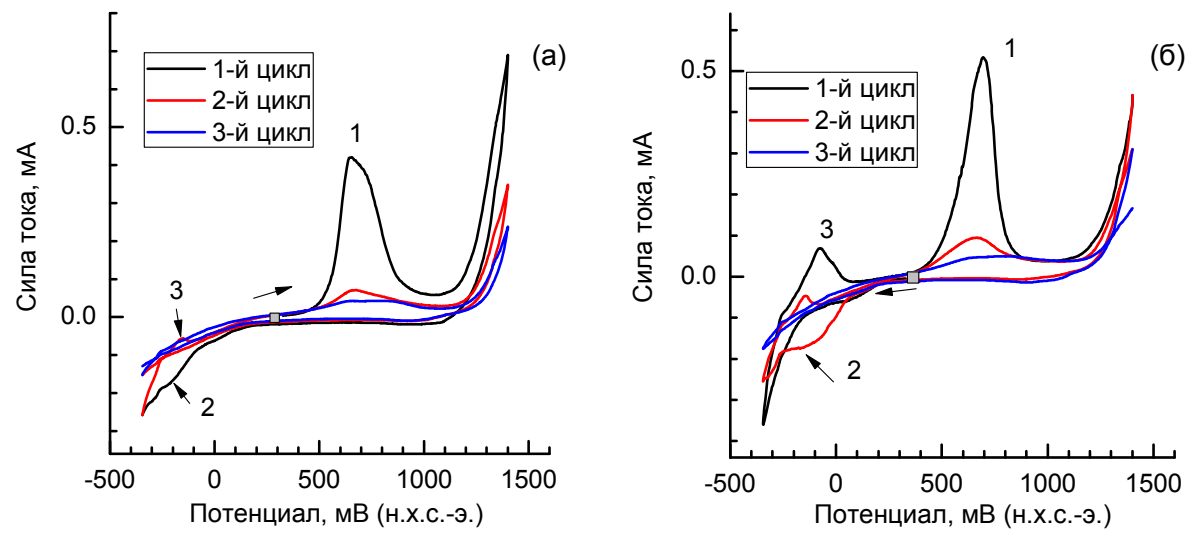

Рис. 2. Вольтамперные зависимости иммобилизованных на пирографите продуктов взаимодействия $\mathrm{H}_{2} \mathrm{PdCl}_{4}$ с раствором формальдегида в $0,1 \mathrm{M} \mathrm{HCl}$. Скорость развертки потенциала $20 \mathrm{mB} / \mathrm{c}, 22{ }^{\circ} \mathrm{C}$. Направление развертки потенциала: а) анодное и б) катодное

Fig. 2. Current-voltage characteristics of the products of reaction of $\mathrm{H}_{2} \mathrm{PdCl}_{4}$ with formaldehyde solution in $0.1 \mathrm{M}$ $\mathrm{HCl}$ immobilized on pyrographite. The potential sweep speed is $20 \mathrm{mV} / \mathrm{s}, 22{ }^{\circ} \mathrm{C}$. The potential sweep direction: a) anodic and б) cathodic 


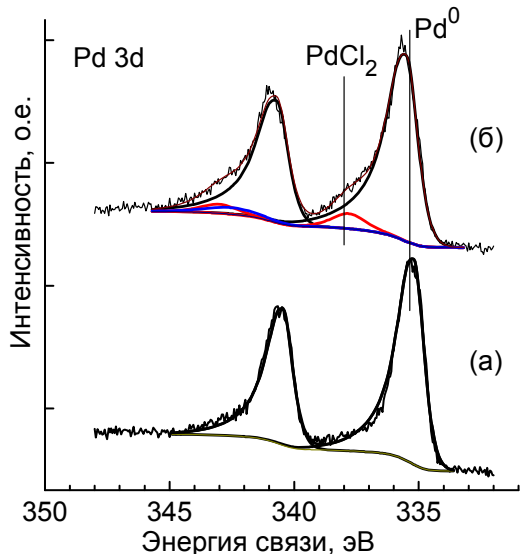

Рис. 3. Рентгеновские фотоэлектронные спектры, линия $\mathrm{Pd} 3 \mathrm{~d}$ частиц палладия полученных формальдегидным восстановлением и осажденных на пирографите, а) - исходные, б) - после окисления в ходе развертки до 600 мВ от компромиссного значения потенциала

Fig. 3. X-ray photoelectron spectra, Pd $3 d$ line of palladium particles obtained by formaldehyde reduction and deposited on pyrographite, a) - initial, б) - after oxidation during sweep to $600 \mathrm{mV}$ from the compromise potential

Пик 2, представляющий собой плечо, в области активного восстановления водорода присутствует только в первом цикле (рис. $2 a$ ), в последующих циклах он не наблюдается. В случае катодного начала развертки пик 2 наблюдается только во втором цикле, поскольку в первом цикле весь палладий находится в металлическом состоянии (рис. 2б). Это подтверждается рентгеновскими фотоэлектронными спектрами - линия Pd 3d подгоняется одной компонентой с энергией связи 335,2 эВ, что соответствует металлическому палладию (рис. 3). В спектре окисленного в ходе развертки потенциала до 600 мВ образца появляется компонента при 337,9 эВ, которая указывает на то, что происходит окисление металлического палладия до комплексных ионов $\mathrm{PdCl}_{4}^{2-}$.

Частицы палладия, полученные формальдегидным восстановлением, имеют электрохимический пик окисления при $660 \mathrm{mB}$, что сопоставимо с частицами, образующимися при катодном восстановлении (реакция 1). Различие частиц состоит лишь в том, что полученный при катодном восстановлении палладий будет содержать в своей решетке водород, который удаляется в условиях эксперимента при катодном потенциале выше -100 - 0 мВ (реакция 2). Окисление и восстановление палладия и отвечающие им пики наблюдаются при различных значениях потенциала ввиду перенапряжения окисления $\mathrm{Pd}^{0}$ на углеродном материале [19, 20].

\section{3. Наночастицы сульфида палладия, соотношение реагентов $S / P d=1: 1$}

На рис. 4 приведены вольтамперные зависимости продуктов взаимодействия $\mathrm{H}_{2} \mathrm{PdCl}_{4} \mathrm{c}$ сульфидом натрия, синтезированных при мольном соотношении реагентов $\mathrm{S} / \mathrm{Pd}=1: 1$. В первом цикле развертки, начатой в анодном направлении, наблюдается двойной пик (пик 1) при 780 и 840 мВ, очевидно, отвечающий за окисление частиц $\mathrm{Pd}(\mathrm{II})-\mathrm{S}$ (рис. $4 a$ ). Наблюдаемая в ряде случаев двойственность и более сложная структура пиков обусловлены тем, что эти процессы 

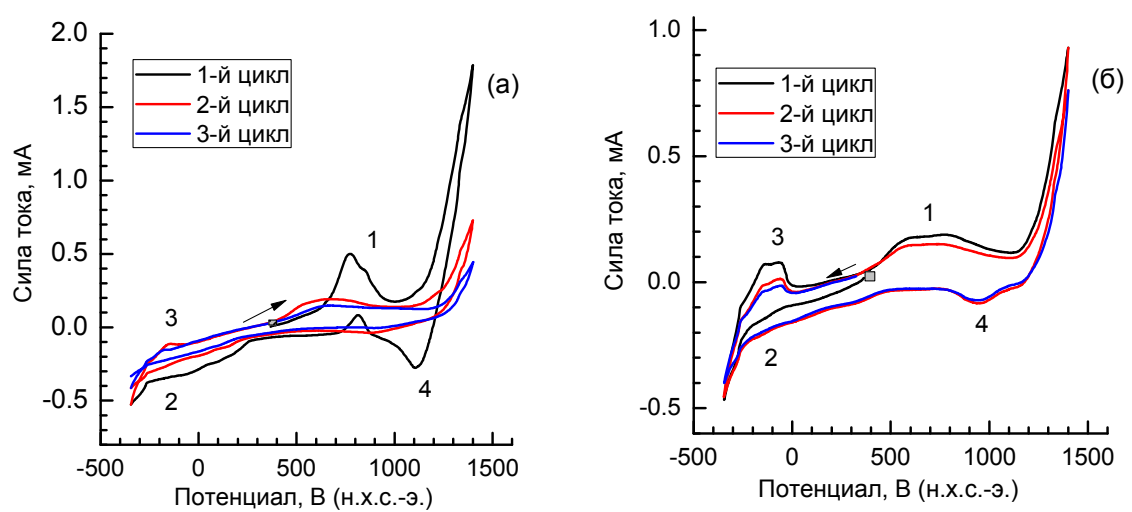

Рис. 4. Вольтамперные зависимости иммобилизованных на пирографите продуктов взаимодействия $\mathrm{H}_{2} \mathrm{PdCl}_{4}$ с сульфидом натрия (соотношение реагентов $\mathrm{S} / \mathrm{Pt}=1$ ), снятые в $0,1 \mathrm{M}$ растворе $\mathrm{HCl}$. Скорость развертки потенциала $20 \mathrm{MB} / \mathrm{c}, 22^{\circ} \mathrm{C}$. Направление развертки потенциала: а) анодное и б) катодное

Fig. 4. Current-voltage characteristics of the $\mathrm{H}_{2} \mathrm{PdCl}_{4}$ interaction with sodium sulfide immobilized on pyrographite with sodium sulfide (reagent ratio $\mathrm{S} / \mathrm{Pt}=1$ ), taken in $0.1 \mathrm{M} \mathrm{HCl}$ solution. The potential sweep speed is $20 \mathrm{mV} / \mathrm{s}$, $22{ }^{\circ} \mathrm{C}$. The potential sweep direction: a) anodic and 6 ) cathodic

протекают в две и более стадий, каждая из которых соответствует переносу одного электрона. Далее, на катодной ветви в области 1000 - 1100 мВ появляется пик (пик 4), не наблюдавшийся в описанных выше системах (рис. $4 a, \sigma$ ). Это можно объяснить наличием в системе серы, в данном случае происходит восстановление продукта ее окисления, образовавшегося при анодном направлении развертки потенциала в области $\mathrm{E}>1200$ мВ. Пик 2, отвечающий восстановлению палладия до металла, слабо выражен, после него начинается резкое возрастание катодного тока (рис. $4 a, \sigma)$, отвечающее восстановлению водорода, по реакции 2.

$$
2 \mathrm{H}^{+}+2 \mathrm{e}^{-} \rightarrow \mathrm{H}_{2}
$$

Часть восстановленного водорода растворяется в палладии, а затем во втором цикле развертки он окисляется по реакции, обратной (2), данному процессу соответствует пик 3 при -200 мВ (рис. 4a). В случае анодного начала развертки потенциала пик 3 значительно ниже, чем в случае с катодным началом развертки (рис. 4б). Очевидно, это связано с тем, что при развертке в катодную область происходит восстановление металла и закрепление его на поверхности электрода, а не окисление частиц $\mathrm{Pd}(\mathrm{II})-\mathrm{S}$ и их переход в раствор, как это случается при развертке в анодном направлении (рис. 4a). При этом интенсивность пика 3 пропорциональна количеству металлического палладия на поверхности, в котором растворяется водород.

\section{4. Наночастицы сульфида палладия, соотночение реагентов $S / P d=3: 1$}

На рис. 5 приведены вольтамперные зависимости продуктов взаимодействия $\mathrm{H}_{2} \mathrm{PdCl}_{4}$ с сульфидом натрия, с мольным соотношением реагентов $-\mathrm{S} / \mathrm{Pd}=3: 1$. В первом цикле развертки потенциала, начатой в анодном направлении, пик 1, отвечающий окислению металлического палладия, в области 800 мВ не наблюдается (рис. $5 a$ ). При этом первый пик на анодной ветви наблюдается в области 1100 - 1150 мВ. В данном случае, он отвечает окислению наночастиц $\mathrm{Pd}(\mathrm{II})-\mathrm{S}$ и переходу $\mathrm{Pd}(\mathrm{II})$ в $\mathrm{Pd}(\mathrm{II})-\mathrm{Cl}$. Сера окисляется, как показывает 

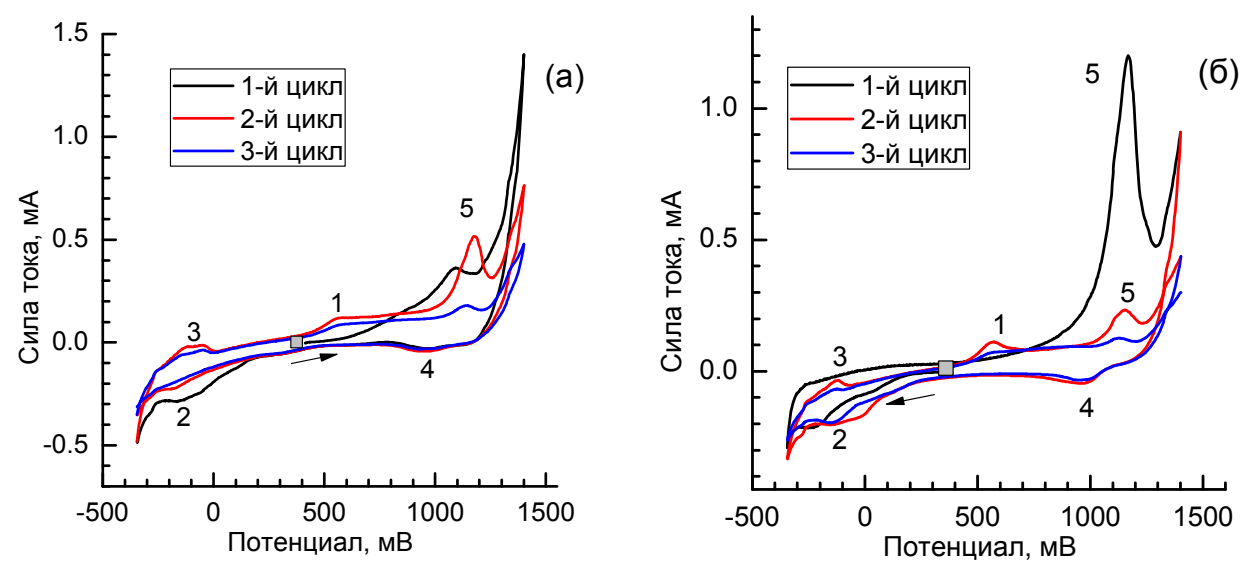

Рис. 5. Вольтамперные зависимости иммобилизованных на пирографите продуктов взаимодействия $\mathrm{H}_{2} \mathrm{PdCl}_{4}$ с сульфидом натрия (соотношение реагентов $\mathrm{S} / \mathrm{Pt}=3$ ), снятые в $0,1 \mathrm{M}$ растворе $\mathrm{HCl}$. Скорость развертки потенциала $20 \mathrm{MB} / \mathrm{c}, 22^{\circ} \mathrm{C}$. Направление развертки потенциала: а) анодное и б) катодное

Fig. 5. Current-voltage characteristics of the $\mathrm{H}_{2} \mathrm{PdCl}_{4}$ interaction with sodium sulfide immobilized on pyrographite with sodium sulfide (reagent ratio $\mathrm{S} / \mathrm{Pt}=3$ ), taken in $0.1 \mathrm{M} \mathrm{HCl}$ solution. The potential sweep speed is $20 \mathrm{mV} / \mathrm{s}$, $22{ }^{\circ} \mathrm{C}$. The potential sweep direction: a) anodic and б) cathodic

РФЭС (рис. 6б), до тиосульфатной и/или сульфатной формы, т.к. в спектрах линии $\mathrm{S} 2 \mathrm{p}$ образца после развертки потенциала до 1400 мВ имеется компонента с энергией связи 168,8 эВ. Данная энергия связи может отвечать как $\mathrm{SO}_{4}{ }^{2-}$, так и $\mathrm{S}_{2} \mathrm{O}_{3}{ }^{2-}$. В спектре присутствует компонента с энергией связи 162,4 эВ. Помимо сульфидной серы она может также соответствовать второму атому серы, входящему в состав $\mathrm{S}_{2} \mathrm{O}_{3}{ }^{2-}$ и не связанному с кислородом (рис. 6б). Далее на катодной ветви появляется пик 4 (при 1000 мВ), как было сказано выше, отвечающий восстановлению окисленных форм серы (рис. $5 a, \sigma)$. Пики, отвечающие процессам восстановления палладия до металла и окислению растворенного в его решетке водорода, в данном случае также наблюдаются (пики 2 и 3 на рис. $5 a$ и 5б). Пик 1, отвечающий окислению $\mathrm{Pd}^{0}$ до $\mathrm{Pd}(\mathrm{II})$, появляется во втором и последующих циклах (рис. $\left.5 a, \sigma\right)$ - после того, как исходные частицы Pd(II)-S были разрушены высоким анодным потенциалом (E = 1400 мB). При этом исходные частицы, по-видимому, слабо восстанавливаются при катодном потенциале, применяемом в условиях эксперимента $(\mathrm{E}=-350 \mathrm{MB})$, и металлический палладий не образуется. На вольтамперных зависимостях частиц сульфида палладия $(\mathrm{S} / \mathrm{Pd}=3)$ во втором и последующих циклах на анодной ветви наблюдается два пика, отвечающих окислению палладийсодержащих частиц (рис. 5). Первый пик, либо плечо, отвечает окислению металлического палладия до $\mathrm{Pd}(\mathrm{II})-\mathrm{S}$ в области 500-600 мВ, в общем виде процесс можно представить уравнением полуреакции:

$$
\mathrm{Pd}^{0}-2 \mathrm{e}^{-}+\mathrm{S}^{2-} \rightarrow \mathrm{PdS}
$$

И второй пик (пик 5, рис. 5) отвечает окислению серы, входящей в состав частиц PdS, например до тиосульфата:

$$
2 \mathrm{PdS}+3 \mathrm{H}_{2} \mathrm{O}+8 \mathrm{Cl}^{-}-8 \mathrm{e}^{-} \rightarrow 2 \mathrm{PdCl}_{4}^{2-}+\mathrm{S}_{2} \mathrm{O}_{3}^{2-}+6 \mathrm{H}^{+} .
$$



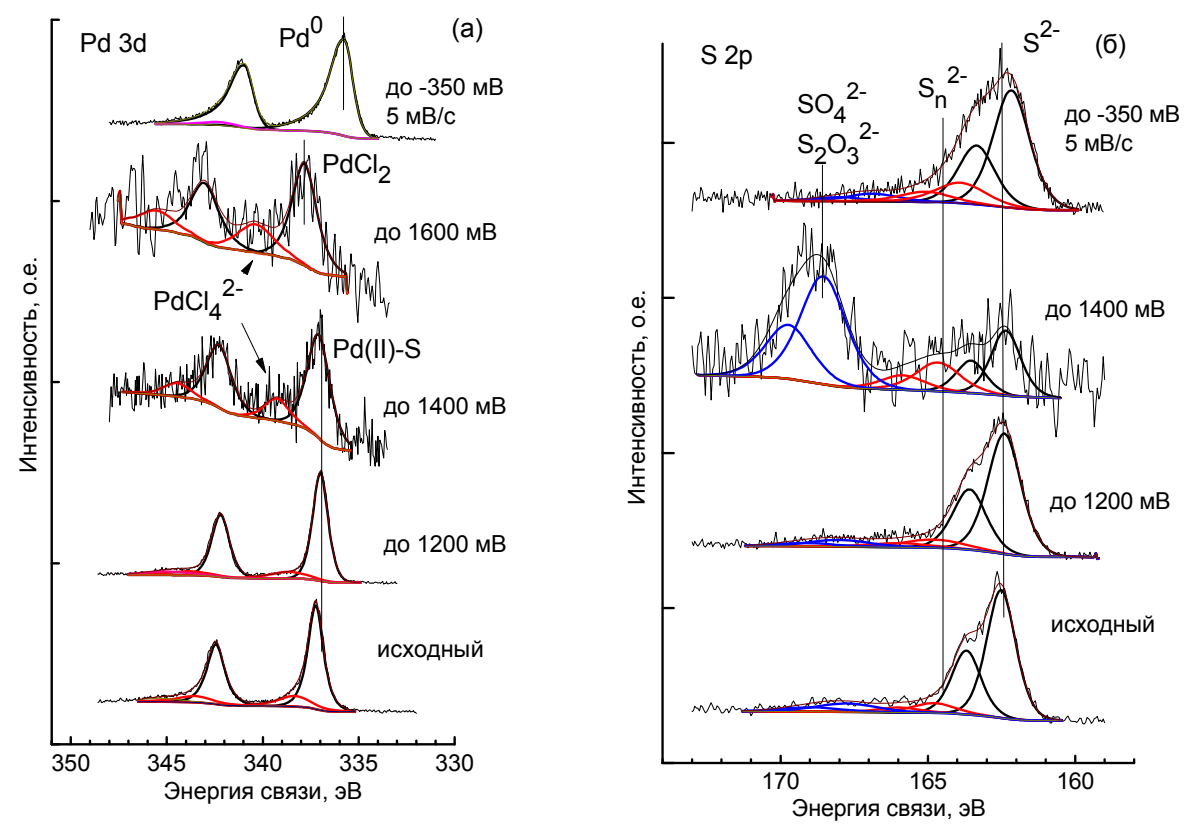

Рис. 6. Рентгеновские фотоэлектронные спектры, линии $\mathrm{Pd} 3 \mathrm{~d}$ (а) и $\mathrm{S} 2 \mathrm{p}$ (б), иммобилизованных на пирографите продуктов взаимодействия $\mathrm{H}_{2} \mathrm{PdCl}_{4}$ с раствором $\mathrm{Na}_{2} \mathrm{~S}$ (соотношение реагентов $\mathrm{S} / \mathrm{Pt}=3: 1$ ), исходные и модифицированные в ходе развертки потенциала до определенного значения в $0,1 \mathrm{M}$ растворе $\mathrm{HCl}$. Скорость развертки потенциала $20 \mathrm{MB} / \mathrm{c}, 22{ }^{\circ} \mathrm{C}$

Fig. 6. X-ray photoelectron spectra, $\mathrm{Pd} 3 \mathrm{~d}$ (a) and $\mathrm{S} 2 \mathrm{p}$ (б) lines, immobilized on pyrographite products of the interaction of $\mathrm{H}_{2} \mathrm{PdCl}_{4}$ with $\mathrm{Na}_{2} \mathrm{~S}$ solution (reagent ratio $\mathrm{S} / \mathrm{Pt}=3$ : 1), initial and modified during potential sweep to a certain values in $0.1 \mathrm{M} \mathrm{HCl}$ solution. Potential sweep rate of $20 \mathrm{mV} / \mathrm{s}, 22{ }^{\circ} \mathrm{C}$

Образующийся тиосульфат (либо другая форма серы) затем при катодном направлении развертки частично восстанавливается до $\mathrm{S}^{2-}$, в данном случае в области 1000 мВ (пик 4). После чего ионы $\mathrm{S}^{2-}$ вновь образуют сульфид палладия.

$$
\mathrm{PdCl}_{4}^{2-}+\mathrm{S}^{2-} \rightarrow \mathrm{PdS}+4 \mathrm{Cl}^{-}
$$

При низкой скорости развертки потенциала -5 мB/с до -350 мB частицы Pd(II)-S успевают восстановиться до металла практически полностью (рис. $6 a$ ). Также на данном образце можно видеть, что сера остается на поверхности пирографита в виде сульфидных групп в адсорбированном состоянии (рис. 6б). В целом данные РФЭС согласуются с данными циклической вольтамперометрии. В том числе можно видеть, что в области от 1200 до 1400 мВ происходит резкое увеличение скорости окисления частиц $\mathrm{Pd}(\mathrm{II})-\mathrm{S}(\mathrm{S} / \mathrm{Pd}=3: 1)$. Палладий и сера удаляются с поверхности (интенсивности линий $\mathrm{Pd} 3 \mathrm{~d}$ и $\mathrm{S} 2 \mathrm{p}$ понижаются на порядок (рис. 6)), при этом сера переходит в окисленные кислородсодержащие формы, а палладий - в раствор в виде хлоридных комплексных ионов. При анодной развертке выше 1600 мВ сера удаляется с поверхности полностью.

Сульфидные наночастицы, полученные при мольном соотношении реагентов $\mathrm{S} / \mathrm{Pd}=1: 1$, окисляются при более низком анодном потенциале, чем полученные при большем мольном соотношении реагентов $-\mathrm{S} / \mathrm{Pd}=3: 1$, в первом случае электрохимический пик окисления ча-

$$
-50-
$$


стиц лежит в области 800 мВ, а во втором - вблизи потенциала 1200 мВ. Также наночастицы $\mathrm{Pd}(\mathrm{II})-\mathrm{S}(\mathrm{S} / \mathrm{Pd}=1: 1)$ менее устойчивы к катодным потенциалам, поскольку после развертки потенциала до -350 мВ эти частицы разрушаются. Далее, при развертке потенциала на анодной ветви наблюдается не соответствующий пик окисления этих частиц при $800 \mathrm{mB}$, а пик при 650 эВ, отвечающий окислению металлического палладия. Соединения серы в данном случае переходят в раствор либо удаляются из системы в виде $\mathrm{H}_{2} \mathrm{~S}$, и дальнейшее электрохимическое поведение данной системы схоже с металлическими наночастицами палладия, образующимися спонтанно при контакте пиролитического графита с раствором $\mathrm{H}_{2} \mathrm{PdCl}_{4}$. Такое различие в электрохимическом поведении частиц сульфида палладия, полученных при разных мольных соотношениях реагентов (S/Pd), не может быть обусловлено размерным эффектом. Наиболее вероятным объяснением может являться различие в стехиометрии сульфида палладия, поскольку существуют серадефицитные сульфиды палладия, например [21, 22] $\mathrm{Pd}_{4} \mathrm{~S}$, $\mathrm{Pd}_{3} \mathrm{~S}, \mathrm{Pd}_{16} \mathrm{~S}_{7}$, которые образуются при низких мольных соотношениях $\mathrm{S} / \mathrm{Pd}$ и различить которые методом РФЭС весьма сложно, поскольку различие в энергиях связи составляет десятые доли эВ [23].

Системы Pd-(S), полученные при низких мольных соотношениях ( $\mathrm{S} / \mathrm{Pd}=1)$, не только менее устойчивы к окислительно-восстановительному воздействию, но и необратимо разрушаются при высоких катодных либо анодных потенциалах. После окисления при высоких анодных потенциалах наночастиц сульфида палладия, полученных при высоких мольных соотношениях $(\mathrm{S} / \mathrm{Pd}=3)$, происходит переход палладия и серы в виде окисленных форм в раствор. Но при понижении потенциала на электроде, на его поверхности снова образуются палладий в сульфидной, а не металлической форме, как в случае низких мольных соотношений. Данное заключение следует из того, что пик, отвечающий окислению частиц Pd(II)-S при 1200 мB, имеется во втором и последующих циклах.

\section{Заключение}

Методами циклической вольтамперометрии и РФЭС показаны различия в электрохимическом поведении металлических и сульфидных наночастиц палладия различного состава на пирографитном электроде. Металлические частицы диаметром 20 нм в 0,1 M HCl стабильны в области электрохимического потенциала до $600 \mathrm{MB}$. Выше $600 \mathrm{mB}$ происходит окисление палладия до $\mathrm{PdCl}_{4}^{2-}$. Частицы $\mathrm{Pd}(\mathrm{II})-\mathrm{S}$, полученные при мольном соотношении реагентов $\mathrm{S} /$ $\mathrm{Pd}=1: 1$, менее устойчивы к воздействию окисления или восстановления, чем частицы $\mathrm{Pd}(\mathrm{II})-\mathrm{S}$ $(\mathrm{S} / \mathrm{Pd}=3: 1)$. В первом случае частицы необратимо окисляются при потенциале выше 750 мВ и восстанавливаются до металла при потенциалах ниже -100 мВ. Во втором случае окисление происходит при потенциале выше $1100 \mathrm{mB}$, восстановление также при потенциале ниже -100 мВ, но медленнее, чем в случае с соотношением $\mathrm{S} / \mathrm{Pd}=1: 1$.

\section{Благодарности}

Исследование выполнено при финансовой поддержке Российского фонда фундаментальных исследований, Правительства Красноярского края, Красноярского краевого фонда поддержки научной и научно-технической деятельности в рамках научного проекта № 17-45240759 p_a. 


\section{Список литературы}

1. Zhou W.P., Lewera A., Larsen R., Masel R.I., Bagus P.S., Wieckowski A. Size effects in electronic and catalytic properties of unsupported palladium nanoparticles in electrooxidation of formic acid. The Journal of Physical Chemistry B 2006. Vol. 110(27), P. 13393-13398.

2. Teranishi T., Miyake M. Size control of palladium nanoparticles and their crystal structures. Chemistry of Materials 1998. Vol. 10(2), P. 594-600.

3. Narayanan R., El-Sayed M.A. Effect of catalysis on the stability of metallic nanoparticles: Suzuki reaction catalyzed by PVP-palladium nanoparticles. Journal of the American Chemical Society 2003. Vol. 125(27), P. 8340-8347.

4. Semagina N., Renken A., Kiwi-Minsker L. Palladium nanoparticle size effect in 1-hexyne selective hydrogenation. The Journal of Physical Chemistry C 2007. Vol. 111(37), P. 13933-13937.

5. Gammons C.H., Bloom M.S., Yu Y. Experimental investigation of the hydrothermal geochemistry of platinum and palladium: I. Solubility of platinum and palladium sulfide minerals in $\mathrm{NaCl} / \mathrm{H}_{2} \mathrm{SO}_{4}$ solutions at $300{ }^{\circ} \mathrm{C}$. Geochimica et Cosmochimica Acta 1992. Vol. 56, P. 3881-3894.

6. Gammons C.H., Bloom M.S. Experimental investigation of the hydrothermal geochemistry of platinum and palladium: II. The solubility of PtS and PdS in aqueous sulfide solutions to $300{ }^{\circ} \mathrm{C}$. Geochimica et Cosmochimica Acta 1992. Vol. 57, P. 2451-2467.

7. Mwase J.M., Petersen J., Eksteen J.J. A conceptual flowsheet for heap leaching of platinum group metals (PGMs) from a low-grade ore concentrate. Hydrometallurgy 2012. Vol. 111(112), P. $129-135$.

8. Wickenden A.E., Krause R.A. Polysulfide Chelates. Desulfuration of $\mathrm{PtS}_{15}{ }^{2-}$ and the Synthesis of $\mathrm{PtS}_{10}{ }^{2-}$. Inorganic Chemistry 1968. Vol. 8, P. 779-783.

9. Muller A., Diemann E. Polysulfide complexes of metals. Advances in Inorganic Chemistry 1987. Vol. 31, P. 89-122.

10. Zamborini F.P., Gross S.M., Murray R.W. Synthesis, characterization, reactivity, and electrochemistry of palladium monolayer protected clusters. Langmuir 2001. Vol. 17(2), P. 481488.

11. Diculescu V.C., Chiorcea-Paquim A.M., Corduneanu O., Oliveira-Brett A.M. Palladium nanoparticles and nanowires deposited electrochemically: AFM and electrochemical characterization. Journal of Solid State Electrochemistry 2007. Vol. 11(7), P. 887-898.

12. Shendage S.S., Patil U.B., Nagarkar J.M. Electrochemical synthesis and characterization of palladium nanoparticles on nafion-graphene support and its application for Suzuki coupling reaction. Tetrahedron Letters 2013. Vol. 54(26), P. 3457-3461.

13. Shao M., Odell J.H., Choi S., Xia Y. Electrochemical surface area measurements of platinumand palladium-based nanoparticles. Electrochemistry Communications 2013. Vol. 31, P. 46-48.

14. De Long H.C., Wilkes J.S., Carlin R.T. Electrodeposition of palladium and adsorption of palladium chloride onto solid electrodes from room temperature molten salts. Journal of the Electrochemical Society 1994. Vol. 141(4), P. 1000-1005.

15. Szabó S., Nagy F. Deposition of Palladium on Platinum in Hydrochloric Acid Media. Israel Journal of Chemistry 1979. Vol. 18(1-2), P. 162-165.

16. Rao C.R.K., Trivedi D.C. Chemical and electrochemical depositions of platinum group metals and their applications. Coordination Chemistry Reviews 2005. Vol. 249(5-6), P. 613-631. 
17. Devanathan M.A.V., Stachurski Z. The adsorption and diffusion of electrolytic hydrogen in palladium. Proceedings of the Royal Society A 1962. Vol. 270(1340), P. 90-102.

18. Simonov P.A., Romanenko A.V., Prosvirin I.P., Moroz E.M., Boronin A.I., Chuvilin A.L., Likholobov V.A. On the nature of the interaction of $\mathrm{H}_{2} \mathrm{PdCl}_{4}$ with the surface of graphite-like carbon materials. Carbon 1997. Vol. 35(1), P. 73-82.

19. Gabrielli C., Grand P.P., Lasia A., Perrot H. Investigation of hydrogen adsorption and absorption in palladium thin films II. Cyclic voltammetry. Journal of the Electrochemical Society 2004. Vol. 150(11), P. A1937-A1942.

20. Hoare J.P. Oxygen overvoltage on bright palladium in acid solutions. Journal of the Electrochemical Society 1965. Vol. 112(11), P. 1129-1133.

21. Diaz-Chao P., Ferrer I.J., Ares J.R., Sanchez C. Cubic $\operatorname{Pd}_{16} \mathrm{~S}_{7}$ as a precursor phase in the formation of tetragonal PdS by sulfuration of Pd thin films. The Journal of Physical Chemistry C 2009. Vol. 113(43), P. 5329-5335.

22. Miller J.B., Alfonso D.R., Howard B.H., O'Brien C.P., Morreale B.D. Hydrogen dissociation on $\mathrm{Pd}_{4} \mathrm{~S}$ surfaces. The Journal of Physical Chemistry C 2009. Vol. 113(43), P. 18800-18806.

23. Romanchenko A.S., Mikhlin Yu.L. An XPS study of products formed on pyrite and pyrrhotine by reacting with palladium(II) chloride solutions. Journal of Structural Chemistry 2015. Vol. 56(3), P. 531-537. 